\title{
Factores ambientales que afectan algunas características postdestete de las cerdas en el trópico de México
}

\author{
Environmental factors affecting some reproductive traits of sows \\ in the tropics of Mexico \\ JE Ek-Mex*, JC Segura-Correa, A Alzina-López, R Aké-López \\ Campus de Ciencias Biológicas y Agropecuarias, Universidad Autónoma de Yucatán, \\ Mérida, Yucatán, México.
}

\begin{abstract}
SUMMARY
The aim of this study was to determine the effect of some environmental factors on weaning to first service interval (WSI), weaning to conception interval (WCI), farrowing to first service interval (FSI), and farrowing interval (FI) and estimate their repeatabilities under the humid tropic conditions of Mexico. More than 33.000 observations from approximately 8.500 sows were used. The statistical model for those traits included the fixed effects of farm $(1,2,3,4)$, farrowing year (2007-2012), farrowing season (dry, rainy and windy), parity number $(1,2, \ldots,>8)$, and significant simple interactions, plus the random effects of sow and the error term. Covariates used were lactation length (LL) and litter size. The means for WSI, WCI, FSI and FI were 6.84 days, 9.80 days, 29.1 days, and 146 days, respectively. All main factors and the year by season interaction had significant effects $(\mathrm{P}<0,05)$ on all response variables, except season for WSI and FSI. Lactation length had a quadratic effect on all variables. The repeatability estimates for WSI, WCI, FSI and FI were $0.067,0.055,0.067$, and 0.050 , respectively. In conclusion, management and feeding programs should consider parity number and duration of lactation in order to improve the reproductive performance of sows. The repeatabilities found for the studied traits were low.
\end{abstract}

Key words: parity, season, reproductive performance, tropics.

\section{RESUMEN}

El objetivo de este estudio fue determinar el efecto de algunos factores ambientales sobre el intervalo destete-primer servicio (IDS), intervalo destete-concepción (IDC), intervalo parto-primer servicio (IPS) e intervalo entre partos (IEP) y estimar sus repetibilidades bajo condiciones del trópico subhúmedo de México. Se utilizaron más de 33.000 observaciones de aproximadamente 8.500 cerdas. El modelo estadístico que describió las variables de respuesta incluyó los efectos de granja (1, 2, 3, 4), año de parto (2007-2012), época de parto (sequía, lluviosa y nortes), número de partos (1 a > 8 ), sus interacciones simples, los efectos aleatorios de cerda y el error. Las covariables usadas fueron duración de la lactación y tamaño de la camada. Las medias para IDS, IDC, IPS, e IEP fueron 6,84, 9,8, 29,1 y 146 días, respectivamente. Todos los factores y la interacción año y época fueron significativas $(\mathrm{P}<0,05)$, con excepción de la época sobre IDS e IPS. La duración de la lactancia tuvo efecto cuadrático sobre todas las variables. Las repetibilidades estimadas para IDS, IDC, IPS, e IEP fueron 0,067, 0,055, 0,067, y 0,05, respectivamente. En conclusión, en los programas de manejo y alimentación deben ser considerados el número de parto y la duración de la lactación para mejorar el comportamiento reproductivo de las cerdas. Las repetibilidades para las características estudiadas fueron bajas.

Palabras clave: parto, época, comportamiento reproductivo, trópico.

\section{INTRODUCCIÓN}

Las características reproductivas afectan el potencial productivo de las cerdas en granjas, en cualquier sistema de producción. La eficiencia en la reproducción de las cerdas se puede cuantificar por medio del intervalo desteteprimer servicio (IDS), intervalo destete a la concepción (IDC), intervalo parto-primer servicio (IPS) e intervalo entre partos (IEP). Cuando estos intervalos son cortos contribuyen a mejorar la eficiencia reproductiva y la acumulación de un menor número de días no productivos; obteniéndose un mayor número de camadas y lechones

Aceptado: 19.06.2014.

* Km 15.5 carretera Mérida-Xmatkuil, Mérida, Yucatán, México; jeemvz@hotmail.com destetados por hembra al año (Vogrin y Škorjanc 2008). Estas características reproductivas pueden utilizarse indirectamente como una medida de manejo de la lactancia, así como indicador de la fertilidad de las cerdas postdestete. Las características reproductivas de las cerdas pueden ser afectadas por la duración de la lactancia, número de parto, tamaño de la camada, época de parto, la exposición al semental después del destete, la raza, el tiempo de la ovulación, las enfermedades, las prácticas de manejo, la nutrición en la lactancia, la temperatura, el fotoperíodo, el estrés y la edad de la cerda (Almond y col 2006, Vogrin y Škorjanc, 2008, Leite y col 2011). La estimación de la repetibilidad es útil para la toma de decisiones sobre la eliminación o no de las cerdas, debido a que conociendo la repetibilidad se puede predecir la habilidad más probable de un animal. 
La identificación de los factores que afectan las características reproductivas es fundamental para adaptar el manejo, las técnicas y tecnologías de los sistemas de producción. Además, su conocimiento es importante para lograr índices óptimos de producción, para incrementar la producción y la productividad de la cerda durante su estancia en la piara reproductora. En México existen algunos estudios acerca de los factores ambientales que afectan las características reproductivas de las cerdas y sus repetibilidades (Segura y Segura 1991, Segura y col $2013^{\mathrm{a}}$, Segura y col 2013 ${ }^{\mathrm{b}}$ ). De acuerdo con los autores, este estudio sería el primero referente a factores ambientales que afectan IDS, IDC e IPS y sus repetibilidades bajo condiciones de trópico de México. Por tanto el objetivo de este estudio fue determinar el efecto de algunos factores ambientales sobre el IDS, IDC, IPS e IEP y estimar sus repetibilidades en cerdas manejadas bajo las condiciones del trópico subhúmedo de México.

\section{MATERIAL Y MÉTODOS}

Se utilizaron los datos de cuatro granjas comerciales del estado de Yucatán, México. El estado se localiza entre los $19^{\circ} 30^{\prime}$ y $21^{\circ} 35^{\prime}$ latitud norte y $90^{\circ} 24^{\prime}$ longitud oeste. El clima de la región es tropical subhúmedo, con temperatura media de $26^{\circ} \mathrm{C}$, precipitación media de $1.100 \mathrm{~mm}$ y humedad relativa de $78 \%$ (INEGI 2004).

Las granjas 1, 2 y 4 eran granjas de ciclo completo con $3.900,1.200$ y 550 cerdas, respectivamente. La granja 3 era una granja de dos sitios (cría y producción) con 320 cerdas. En todas las granjas las cerdas fueron de la línea genética comercial Camborough 22, producían sus propios reemplazos y practicaban la cuarentena de las hembras. En las cuatro granjas, el servicio se llevó a cabo utilizando la inseminación artificial y posteriormente la monta natural. La detección de estros se realizó dos veces al día (6:00 y 18:00 horas) por medio de un semental y presionando el lomo de las cerdas para observar el reflejo de parada. Las inseminaciones se realizaron dos veces por día, una en la mañana y otra por la tarde (12 a 18 horas), después de detectado el estro, y cada cerda recibió tres dosis de semen. La condición corporal de las cerdas al parto fue entre 3 y 4 . La transferencia o adopción de lechones se realizaba en los dos primeros días después del nacimiento. Las cerdas se alimentaron con dietas comerciales de acuerdo con la etapa productiva. Las cerdas jóvenes (aproximadamente $200 \mathrm{~kg}$ de peso corporal) recibieron 2,6 kg de alimento/día con 3.000 kcal EM / kg, $16 \%$ de proteína bruta y $0,8 \%$ de lisina; mientras que las hembras con $300 \mathrm{~kg}$ de peso corporal recibieron $3,2 \mathrm{~kg}$ de alimento/día.

Los datos se obtuvieron de los registros de producción de 2007 a 2012 capturados en el programa PigCHAMP®. La información que se utilizó fue la identificación de la granja y de la cerda, número de parto, fecha de parto y de destete, y tamaño de la camada al nacer.

\section{ANÁLISIS ESTADÍSTICO}

El modelo estadístico que describió las variables de respuesta: IDS, IPS, IDC e IEP incluyó los efectos fijos de granja $(1,2,3,4)$, año de parto (2007 a 2012), época de parto (seca de febrero a mayo, lluviosa de junio a septiembre y nortes de octubre a enero), número de parto $(1,2,3,4$, $5,6,7,>8)$, las interacciones simples significativas y los efectos aleatorios de cerda $(0, \sigma 2 c)$ y el error $\operatorname{NID}(0, \sigma 2 e)$. Asimismo, en el modelo se utilizaron como covariables el efecto lineal y cuadrático de la duración de la lactación y el efecto lineal de tamaño de la camada (número de lechones nacidos vivos). El análisis estadístico se realizó mediante el procedimiento MIXED (SAS 2008). El nivel de significancia para los análisis usados fue de $\mathrm{P}<0,05$. Las estimaciones de repetibilidad y los errores estándar se calcularon usando los componentes de varianza entre y dentro de cerdas producidos por el procedimiento MIXED (SAS 2008), de acuerdo con las fórmulas proporcionadas por Becker (1992).

\section{RESULTADOS}

Las medias y las desviaciones estándares para IDS, IDC, IPS e IEP fueron 6,84 6 , 7 días, 9,8 $8 \pm 12,1$ días, $29,1 \pm 7,19$ días y $146,0 \pm 12,4$ días, respectivamente. Se encontró efecto significativo para IDS e IPS de granja $(\mathrm{P}<0,0001)$, año $(\mathrm{P}<0,0001)$ y número de parto $(\mathrm{P}<0,0001)$. Los factores significativos para las variables de respuesta IDC fueron granja $(\mathrm{P}<0,0001)$, año $(\mathrm{P}<0,0001)$, época de parto $(\mathrm{P}<0,0042)$, número de parto $(\mathrm{P}<0,0001)$, la interacción de año por época de parto $(\mathrm{P}<0,0035)$ y la interacción de granja por época de parto $(\mathrm{P}<0,047)$. Se encontró efecto significativo para IEP de granja $(\mathrm{P}<0,0001)$, año $(\mathrm{P}<0,0001)$, época de parto $(\mathrm{P}<0,0042)$, número de parto $(\mathrm{P}<0,0001)$, y la interacción de año por época de parto $(\mathrm{P}<0,0018)$. Las medias de cuadrados mínimos generalizados por factor para las variables de respuesta se muestran en el cuadro 1.

En la figura 1 se observa la interacción entre el año de parto y la época de parto de todas las variables de respuesta. En general se observa que los efectos de época variaron con los años; en unos años una época fue mejor y en otros años otra época. Asimimo, se observa una disminución de las medias de todos los intervalos según el transcurso de los años de estudio a partir del 2008. El peor comportamiento reproductivo correspondió a la granja 4 . La interacción mostró un efecto diferente de las épocas según la granja para IDC.

El efecto lineal y cuadrático de duración de la lactación (DL) fue significativo $(\mathrm{P}<0,0001)$ en todas las variables de respuesta. La media y desviación estándar de la DL fueron 22,4 $\pm 2,9$ días. La figura 2 muestra la relación del IDS e IDC con DL. Los menores IDS correspondieron a las cerdas que se destetaron entre los días 19 y 27 de lactación, y el menor IDS fue de 6,5 días. Los menores 
Cuadro 1. Medias de cuadrados mínimos y errores estándar por fuente de variación para el intervalo destete-primer servicio (IDS), intervalo parto-primer servicio (IPS), intervalo destete a la concepción (IDC) e intervalo entre partos (IEP) de cerdas en el trópico subhúmedo de México.

Least squares means and standard errors by source of variation for the interval from weaning to first service (IDS), interval from calving to first service (IPS), weaning to conception interval (IDC) and farrowing interval (IEP) of sows in the tropics sub-humid of Mexico.

\begin{tabular}{lccccccc}
\hline Fuente de variación & $\mathrm{n}$ & IDS & IPS & $\mathrm{n}$ & IDC & $\mathrm{n}$ & IEP \\
\hline Granja & & & & & & & \\
1 & 24.084 & $6,3 \pm 0,07^{\mathrm{b}}$ & $28,7 \pm 0,07^{\mathrm{b}}$ & 23.35 & $8,46 \pm 0,18^{\mathrm{d}}$ & 23.721 & $145,6 \pm 0,17^{\mathrm{b}}$ \\
2 & 7.018 & $6,1 \pm 0,09^{\mathrm{c}}$ & $28,5 \pm 0,09^{\mathrm{c}}$ & 4.576 & $9,84 \pm 0,21^{\mathrm{c}}$ & 6.094 & $145,2 \pm 0,19^{\mathrm{b}}$ \\
3 & 2.987 & $6,3 \pm 0,13^{\mathrm{bc}}$ & $28,7 \pm 0,13 \mathrm{~b}^{\mathrm{c}}$ & 2.389 & $11,9 \pm 0.28^{\mathrm{b}}$ & 2.783 & $147,8 \pm 0,26^{\mathrm{a}}$ \\
4 & 4.304 & $8,45 \pm 0,11^{\mathrm{a}}$ & $30,82 \pm 0,11^{\mathrm{a}}$ & 3.214 & $12,5 \pm 0,25^{\mathrm{a}}$ & 3.917 & $148,0 \pm 0,23^{\mathrm{a}}$ \\
Época de parto & & & & & & & \\
Sequía & 12.787 & $6,8 \pm 0,08^{\mathrm{a}}$ & $29,17 \pm 0,07^{\mathrm{a}}$ & 11.536 & $10,41 \pm 0,2^{\mathrm{b}}$ & 12.631 & $146,3 \pm 0,16^{\mathrm{b}}$ \\
Lluviosa & 13.538 & $6,76 \pm 0,11^{\mathrm{a}}$ & $29,13 \pm 0,07^{\mathrm{a}}$ & 11.498 & $11,13 \pm 0,2^{\mathrm{a}}$ & 12.409 & $147,19 \pm 0,17^{\mathrm{a}}$ \\
Nortes & 12.068 & $6,81 \pm 0,07^{\mathrm{a}}$ & $29,18 \pm 0,02^{\mathrm{a}}$ & 10.495 & $10,49 \pm 0,21^{\mathrm{b}}$ & 11.475 & $146,17 \pm 0,17^{\mathrm{b}}$ \\
Número de parto & & & & & & & \\
1 & & & & & & & \\
2 & 9.932 & $8,11 \pm 0,07^{\mathrm{a}}$ & $30,48 \pm 0,07^{\mathrm{a}}$ & 9.858 & $13,03 \pm 0,15^{\mathrm{a}}$ & 10.434 & $149,17 \pm 0,14^{\mathrm{a}}$ \\
3 & 8.357 & $6,73 \pm 0,08^{\mathrm{c}}$ & $29,1 \pm 0,08^{\mathrm{bc}}$ & 7.847 & $10,49 \pm 0,16^{\mathrm{b}}$ & 8.498 & $146,52 \pm 0,15^{\text {bef }}$ \\
4 & 6.75 & $7,07 \pm 0,08^{\mathrm{b}}$ & $29,11 \pm 0,08^{\mathrm{b}}$ & 6.066 & $10,77 \pm 0,17^{\mathrm{b}}$ & 6.622 & $146,79 \pm 0,16^{\mathrm{b}}$ \\
5 & 5.44 & $6,86 \pm 0,09^{\mathrm{bc}}$ & $29,23 \pm 0,09^{\mathrm{bc}}$ & 4.74 & $10,64 \pm 0.19^{\mathrm{bc}}$ & 5.131 & $146,62 \pm 0,18^{\mathrm{bc}}$ \\
6 & 4.217 & $6,72 \pm 0,10^{\mathrm{cd}}$ & $28,09 \pm 0,1^{\mathrm{cd}}$ & 3.408 & $10,47 \pm 0,22^{\mathrm{be}}$ & 3.75 & $146,32 \pm 0,21^{\text {cf }}$ \\
7 & 1.542 & $6,39 \pm 0,16^{\mathrm{de}}$ & $28,75 \pm 0,16^{\mathrm{de}}$ & 815 & $9,54 \pm 0,42^{\mathrm{f}}$ & 1.045 & $145,48 \pm 0,38^{\mathrm{eg}}$ \\
$>8$ & 1.005 & $6,25 \pm 0,20^{\mathrm{e}}$ & $28,62 \pm 0,20^{\mathrm{e}}$ & 435 & $9,91 \pm 0,58^{\mathrm{bf}}$ & 573 & $145,63 \pm 0.53^{\text {cde }}$ \\
\hline
\end{tabular}

a,b,c Diferente literal en la columna indica diferencia significativa $(\mathrm{P}<0,05)$.

IDC se encontraron entre los 19 y 30 días de lactación, y el menor IDC fue 11 días. Asimismo se encontró efecto lineal del tamaño de camada $(\mathrm{P}<0,005)$ sobre IDC e IEP con un coeficiente de regresión y error estándar de $-0.063 \pm 0.02$ y $-0.061 \pm 0.02$, respectivamente.

Las estimaciones de repetibilidad y errores estándar de IDS, IPS, IDC e IEP fueron 0,067 $\pm 0,005,0,067 \pm 0,005$, $0,055 \pm 0,004$ y $0,050 \pm 0,004$, respectivamente.

\section{DISCUSIÓN}

La media del IDS encontrada en este estudio (6,84 días) es similar a la reportada por Chansomboon y col (2010) en Tailandia (6,52 días), pero menor a la que encontraron Karveliené y col (2008) en Lituania y Segura y col (2013a) en la región central de México con 9,3 y 7,9 días, respectivamente. Hoshino y Koketsu (2008) reportan 82\% de cerdas con un IDS entre 4-6 días, estas obtuvieron la mayor tasa de partos en comparación con los otros grupos y el mayor número de lechones nacidos vivos comparado con el grupo de 7-20 días de IDS. Estas diferencias de las medias se deben principalmente a diferencias en las condiciones climáticas, diferencias de manejo, líneas genéticas usadas y diferentes condiciones sanitarias entre granjas o regiones. Sin embargo, las medias de IDS indicadas anteriormente son inferiores a las reportadas para el IDC en este estudio, lo que se debe en parte a que el IDC se refiere al número de días que transcurren desde el destete al servicio en donde se logra la concepción, por lo que considera a las cerdas repetidoras de estro, y el IDS se refiere al número de días entre el destete y la presentación del primer estro con inseminación. Se destaca que el IDS e IDC son considerados como los días no productivos, debido a que la cerda durante este período no se encuentra gestando o lactando, pero sí se encuentra generando un gasto de mantenimiento, por ello, si se identifican y se consigue reducirlos se logra incrementar el rendimiento de las granjas. Asimismo, la proximidad de las medias de IDS e IDC indica que la mayoría de las cerdas se preñan al primer servicio.

La media del IDC es similar a los reportados por Gourdine y col (2006) en una estación experimental en Guadalupe de clima tropical y a lo reportado en granjas comerciales en clima templado por Koketsu y Dial (1998) en EE.UU.; sin embargo, es menor al reportado por Tantasuparuk y col (2000) de 18 días en Tailandia. Estas diferencias pueden deberse en parte a las diferentes condiciones climáticas, a las diferencias en la duración de la lactación, así como al consumo y disponibilidad de alimento durante la lactación. Por ejemplo, Koketsu y Dial (1998) reportan una media de DL de 17,4 días, Gourdine y col (2006) reportan una media de 28 días, y Tantasuparuk y col (2000) una media de 27,5 días. También se ha reportado que el incremento en el consumo de alimento de las 

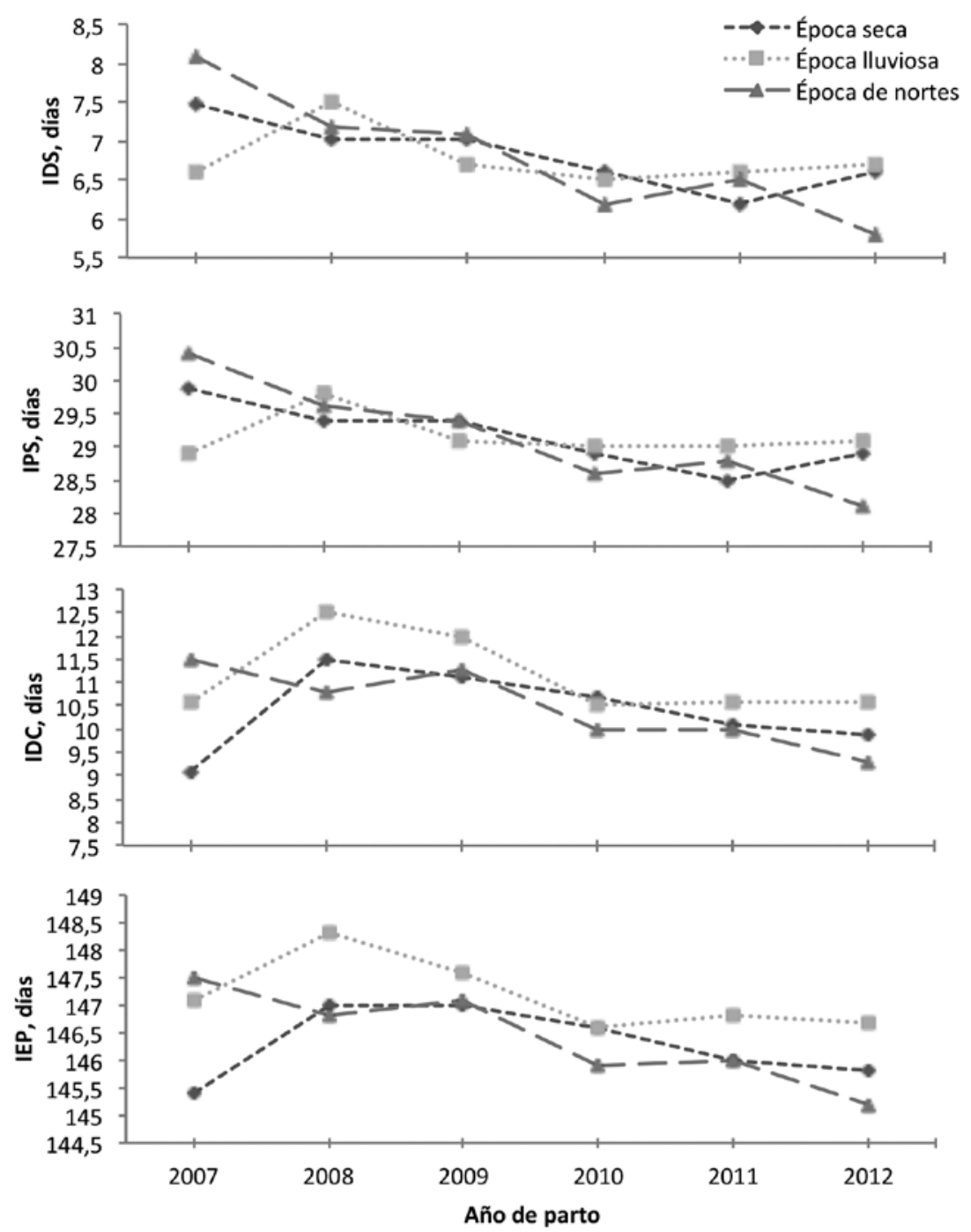

Figura 1. Interacción año de parto por época de parto para: a) intervalo destete al primer servicio (IDS), b) intervalo parto al primer servicio (IPS), c) intervalo destete a la concepción (IDC), d) intervalo entre partos (IEP).

Year by season of farrowing interaction for: a) weaning to first service interval (IDS), b) farrowing to first service interval (IPS), c) weaning to conception interval (IDC) and d) farrowing interval (IEP).

cerdas durante la primera y segunda semana de lactación reduce el retorno del estro después del destete (Koketsu y col 1997). Le Cozler y col (1997) observaron los menores tamaños de camada en las cerdas con un IDC entre 7-10 días, y que el tamaño de camada incrementó al aumentar el IDC de 11 hasta 21 días.

La media del IPS es similar a la media de 29 días reportada por Segura y col $\left(2013^{\mathrm{a}}\right)$, quienes indican que las variables IDS e IPS están asociadas (correlación simple de 0,88 ). Por lo que es posible que existan escasos reportes de IPS y se considere mejor estudiar el IDS, este, como se indicó anteriormente es considerado como parte de los días no productivos que afectan la rentabilidad de las granjas.
La media del IEP de este estudio es inferior al reportado por Segura y Segura (1991) en el trópico húmedo de México y lo encontrado por Tantasuparuk y col (2000) de 175 y 159 días, respectivamente. En contraste, la media del IEP es superior a los 142 días reportado por Díaz y col (2011) en Colombia y lo encontrado por Cavalcante-Neto y col $\left(2009^{\mathrm{a}}\right)$ en Brasil de 141 días. Como ya se mencionó anteriormente, las diferencias en las medias de IEP y las otras variables estudiadas en el presente trabajo, respecto de la literatura, se pudo deber a diferencias en las condiciones climáticas, manejo, líneas genéticas usadas, así como a las diferentes condiciones sanitarias entre granjas y regiones. Otros factores importantes mencionados en la literatura son las diferencias en el manejo de la nutrición 


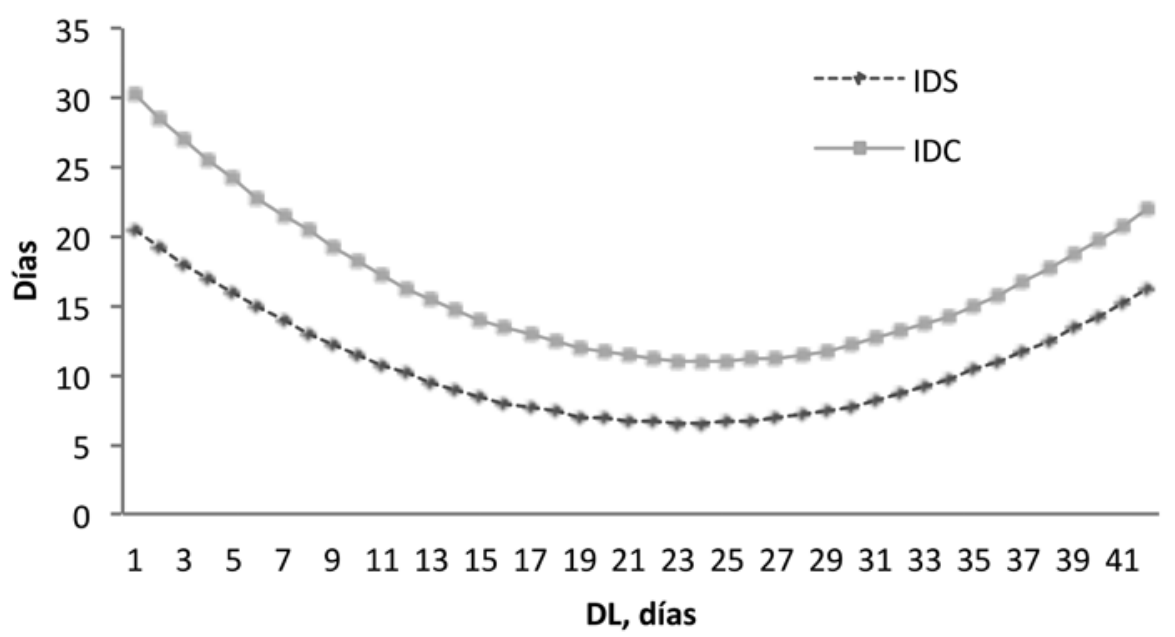

Figura 2. Efecto de duración de la lactación (DL) sobre el intervalo destete primer servicio (IDS) e intervalo destete a la concepción (IDC) de cerdas en el trópico subhúmedo de México.

Effect of lactation length (DL) on the weaning to first service interval (IDS) and weaning to conception interval (IDC) of sows in the sub-humid tropics of Mexico.

durante la lactación, duración de la lactación, el uso o no del semental y capacidad del personal para la detección de celos (Cavalcante-Neto y col 2008, Vogrin y Škorjanc 2008).

El efecto de granja sobre IDS, IDC, IPS e IEP ha sido reportado en otros estudios (Tantasuparuk y col 2000, Leite y col 2011). Diferencias entre las granjas es factible de encontrar debido a los diferentes programas de nutrición, líneas genéticas de las cerdas, tamaño de piara, criterios de eliminación y otras prácticas de manejo aplicado en cada granja. Belstra y col (2004) indican que los factores dentro de cada unidad de producción influyen en la relación inversa del intervalo destete al estro con la duración del estro y el intervalo de la aparición del inicio del estro a la ovulación. De acuerdo con Almond y col (2006), la relación entre el intervalo inicio del estro a la ovulación varía considerablemente en cada granja y entre granjas. Por lo que el comportamiento reproductivo podría diferir considerablemente entre granjas usando idénticos programas de inseminación.

El efecto de la época dependió del año y granja, la época de parto en este estudio no tuvo efecto sobre el IDS e IPS, semejante a lo reportado por Fuentes y col (2000) y Cavalcante-Neto y col (2008). Cavalgante-Neto (2008) y col sugieren que posiblemente cuando las cerdas alcanzan su madurez están menos susceptibles a este efecto, soportando hasta cierto punto el efecto de las diferentes épocas del año y diferencias de manejo. Sin embargo, en este estudio el efecto de la interacción de año por época de parto afectaron a todas las variables de respuesta, semejante a lo reportado por Chansomboon y col (2009) y Segura y col $\left(2013^{a}\right)$. La tendencia a disminuir en las medias de todas las variables de respuesta a partir del 2008, en las diferentes épocas durante los años de estudio, posiblemente se debe a mejores prácticas de manejo, y a cambios de las líneas genéticas usadas en los años de estudio. De acuerdo con Chansomboon y col (2010), el efecto de la interacción es el resultado de la combinación de efectos climáticos (fluctuaciones de temperatura y humedad en las diferentes épocas), nutrición y manejo durante los años de estudio.

La época de parto influyó significativamente sobre el IDC e IEP; resultados similares han sido obtenidos por Tantasuparuk y col (2000) y Lutaaya y col (2009). De acuerdo con Gourdine y col (2004) y Suriyasomboon y col (2006), el efecto de la época de parto se atribuye, en las regiones tropicales, a la alta temperatura ambiente y humedad. El incremento de los días de retorno al celo después del destete durante la época de calor está asociado a la reducción del apetito y a limitaciones de alimento durante la lactación (Prunier y col 1996, Karveliene y col 2008, Leite y col 2011). Además, la elevada temperatura ambiente tiene un efecto directo negativo en el comportamiento reproductivo de las cerdas (Prunier y col 1996).

Efecto de número de parto sobre el IDS, IPS, IDC e IEP ha sido reportado por otros autores (Tantasuparuk y col 2000, Gourdine y col 2006, Schwarz y col 2009). De acuerdo con Suriyasomboon y col (2006) e Imboonta y col (2007), los intervalos más largos en las cerdas de primer parto puede ser debido a un menor consumo voluntario de alimento o pobre nutrición durante la lactación; y como consecuencia de este, las cerdas de primer parto usan sus reservas limitadas de energía y proteína para la producción de leche, mantención y crecimiento en comparación con las cerdas multíparas, lo que causa un retraso en el retorno al celo después del destete (Tummaruk y col 2001, Gourdine y col 2004). Asimismo, un inadecuado programa de inseminación en cerdas primerizas influye en el aumento del IDC e IEP. Se debe considerar que en 
las cerdas de primer parto, el celo dura menos tiempo que las cerdas multíparas (Steverink y col 1999), por lo que el esquema de inseminaciones de las cerdas primerizas no debe ser idéntico al de las cerdas multíparas.

La duración de la DL tuvo un efecto lineal y cuadrático en todas las variables de respuesta de este estudio, lo que concuerda con lo obtenido por Mabry y col (1996), Le Cozler y col (1997), Segura y col $\left(2013^{\mathrm{a}}\right)$. Chansomboon y col (2009) obtuvieron los menores valores de IDS en relación con la DL entre los 26 y 32 días. Sin embargo, Koketsu y Dial (1997) encontraron los menores IDS entre los 17 y 19 días de DL. Le Cozler y col (1997) indican que los menores IDC se obtienen entre 3 y 4 semanas de DL. Asimismo, los menores IDS e IDC, en el presente trabajo, ocurrieron entre los días 19 al 27 y 19 al 30 de lactancia, respectivamente. Lo menores intervalos posiblemente se deban a que con lactaciones largas las cerdas tienen más tiempo para equilibrar su estado metabólico y a no presentar un balance energético negativo, lo que conduce a un menor intervalo de destete al estro, al servicio y a la concepción (Hulten y col 1993, Tummaruk y col 2000, Suriyasomboon y col 2006). También se debe considerar la condición corporal, estado metabólico y nutricional de la cerda durante la lactancia (Hulten y col 1993). Gourdine y col (2006) obtuvieron que las cerdas con intervalos de destete al estro menores de 5 días presentaron un mayor consumo de alimento durante la lactación hasta los 21 días que las cerdas con intervalos de destete al estro mayores de 5 días.

La repetibilidad del IDS es inferior a las repetibilidades calculadas $(0,08$ y 0,14$)$ por Chansomboon y col (2009) en Tailandia y Segura y col $\left(2013^{b}\right)$ en la región central de México. La repetibilidad del IDC es inferior a lo reportado por Le Cozler y col (1997) en cerdas Landrace y Large-White ( 0,11 y 0,13 , respectivamente). Respecto de la repetibilidad del IEP esta es semejante a la encontrada $(0,05-0,07)$ por Cavalcante-Neto y col $\left(2009^{b}\right)$ quienes usaron para su estimación un modelo animal completo. Sin embargo, la repetibilidad aquí estimada es ligeramente mayor a la reportada $(0,03)$ por Segura y col $\left(2013^{b}\right)$ en una granja en Yucatán, México. De acuerdo con Segura y col (2013b), las diferencias en las repetibilidades pudieran deberse a diferencias entre líneas genéticas comerciales, a las condiciones ambientales y de manejo, y a los métodos estadísticos utilizados. Además, los valores bajos de las repetibilidades indican que los efectos ambientales temporales son más importantes que los efectos genéticos. Las repetibilidades bajas enfatizan la importancia de obtener varios registros por cerda para aumentar la exactitud en la predicción de la habilidad más probable y para mejorar la confianza en la predicción de futuros registros de las cerdas en las poblaciones porcinas (Segura y col 2013 ${ }^{\mathrm{b}}$ ).

Se puede concluir que bajo las condiciones del presente estudio todos los factores estudiados fueron fuentes importantes de variación para las variables de respuesta, con excepción de época sobre IDS e IPS. El efecto de granja sugiere que los factores específicos de cada granja influyen en el comportamiento reproductivo postdestete de las cerdas. La reducción de la DL podría ser benéfica para mejorar la rentabilidad de las granjas al incrementarse el número de partos por año. Para mejorar la eficiencia reproductiva de las cerdas, en los programas de producción, se debe considerar el número de parto y la duración de la lactación de la cerda para proporcionarle el manejo y alimentación adecuados. Las repetibilidades bajas de las características postdestete aquí estudiadas indican una mayor influencia del ambiente que aquella debida a la influencia de la genética animal.

\section{REFERENCIAS}

Almond GW, WL Flowers, L Batista, S D‘Allaire. 2006. Diseases of the Reproductive System. In: Diseases of Swine. $9^{\text {th }}$ ed. Iowa State University Press, Ames, USA.

Becker WA. 1992 Repeatability. In: Manual of Quantitative Genetics. $5^{\text {th }}$ ed. Academic Enterprises, Pullman, Washington, USA, Pp 191.

Belstra B A, WL Flowers, MT See. 2004. Factors affecting temporal relationships between estrus and ovulation in commercial sow farms. Anim Reprod Sci 84, 377-394.

Cavalcante-Neto A, JF Lui, JLR Sarmento, MN Ribeiro, JM Costa, H Tonhati. 2008. Fatores ambientais e estimativa de herdabilidade para o intervalo desmame-cio de fêmeas suínas. Rev Bras Zootec 37, 1953-1958.

Cavalcante-Neto A, JF Lui, JLR Sarmento, MN Ribeiro, JMC Monteiro, C Fonseca, H Tonhati. 2009a . Efeitos genéticos e não-genéticos sobre o intervalo de parto em fêmeas suínas no Sudeste do Brasil. Arq Bras Med Vet Zootec 61, 280-285.

Cavalcante-Neto A, JF Lui, JLR Sarmento, MN Ribeiro, JMC Monteiro, C Fonseca, H Tonhati. 2009 ${ }^{\mathrm{b}}$. Estimation models of variance components for farrowing interval in swine. BrazArch Biol Technol $52,69-76$.

Chansomboon C, M Elzo, T Suwanasopee, S Koonawootrittriron. 2009. Genetic and environmental factors affecting weaning-to-first service interval in a Landrace-Large White swine population in Northern Thailand. Kasetsart J (Nat Sci) 43, 669-679.

Chansomboon C, MA Elzo, T Suwanasopee, S Koonawootrittriron. 2010. Estimation of genetic parameters and trends for weaning-to-first service interval and litter traits in a commercial landrace-large white swine population in northern Thailand. Asian-Aust J Anim Sci 23, 543-555.

Díaz C, N Rodríguez, V Vera, G Ramírez, G Casas, J Mogollón. 2011. Caracterización de los sistemas de producción porcina en las principales regiones porcícolas colombianas. Rev Colomb Cienc Реси 24,131-144.

Gourdine JL, D Renaudeau, J Noblet, JP Bidanel. 2004. Effects of season and parity on performance of lactating sows in a tropical climate. Anim Sci 79, 273-282.

Gourdine JL, H Quesnel, JP Bidanel, D Renaudeau. 2006. Effect of Season, Parity and Lactation on Reproductive Performance of Sows in a Tropical Humid Climate. Asian-Aust J Anim Sci 19, 1111-1119.

Hoshino Y, Y Koketsu. 2008. A repeatability assessment of sows mated 4-6 days after weaning in breeding herds. Anim Reprod Sci 108, 22-28.

Hultén F, M Neil, S Einarsson, J Hakansson. 1993. Energy metabolism during late gestation and lactation in multiparous sows in relation to backfat thickness and the interval from weaning to first oestrus. Acta Vet Scand 34, 9-20.

Imboonta N, L Rydhmer, S Tumwasorn. 2007. Genetic parameters and trends for production and reproduction traits in Thai landrace sows. Livest Sci 111, 70-79.

Instituto Nacional de Estadística, Geografía e Informática (INEGI). 2004. Anuario Estadístico de Yucatán. México, D.F. 
Karveliene B, L Serniene, V Riskeviciene. 2008. Effect of different factors on weaning-to-first-service interval in Lithuanian pig herds. Vet Ir Zootechinika 41, 64-69.

Koketsu Y, G Dial. 1997. Factors influencing the postweaning reproductive performance of sows on commercial farms. Theriogenology 47,1445-1461.

Koketsu Y, G Dial, JE Pettigrew, VL King. 1997. Influence of feed intake during individual weeks of lactation on reproductive performance of sows on commercial farms. Livest Prod Sci 49, 217-225.

Koketsu Y, G Dial. 1998. Interactions between the associations of parity, lactation length, and weaning-to-coception interval with subsequent litter size in swine herd using early weaning. Prev Vet Med 37, 113-120.

Le Cozler Y, J Dagorn, JY Dourmad, S Johansen, A Aumatre. 1997. Effect of weaning-to-conception interval and lactation length on subsequent litter size in sows. Liv Prod Sci 51,1-11.

Leite CDS, JF Lui, LG Albuquerque, DN Alves. 2011. Environmental and genetic factors affecting the weaning-estrus interval in sows. Genet Mol Res 10, 2692-2701.

Lutaaya E, A Nakafeero, S Nemaire. 2009. Reproductive performance of two sow lines under arid climatic conditions. South African J Anim Sci 39, 19-23.

Mabry JW, MS Culbertson, D Reeves. 1996. Effects of lactation length on weaning-to-first-service interval, first-service farrowing rate, and subsequent litter size. J Swine Health Prod 4,186-188.

Prunier A, H Quesnel, M Messias de Bragaqa, A Kermabon. 1996. Environmental and seasonal influences on the return-to-oestrusafter weaning in primiparous sows: a review. Lives Prod Sci 45, 103-110.

Schwarz T, J Nowicki, R Tuz. 2009. Reproductive performance of Polish Large White sows in intensive production - effect of parity and season. Ann Anim Sci 9, 269-277.

Segura CJ, CV Segura. 1991. Influencia de algunos factores genéticos y ambientales sobre la eficiencia reproductiva de cerdos en una granja de la Chontalpa, Tabasco. Vet Méx 12, 73-76.
Segura CJ, J Herrera-Camacho, RE Pérez-Sánchez, E Gutiérrez-Vázquez. $2013^{\text {a }}$. Factors affecting and repeatability of some reproductive traits of sows in the central region of Mexico. Rev Colomb Cienc Pecu, en prensa.

Segura CJ, A Alzina, JE Ek, VM Segura. 2013 . Estimadores de índices de constancia para características de la camada al nacimiento e intervalo entre partos de cerdas utilizando aproximaciones de máxima verosimilitud restringida y bayesianas. Revista Científica FCV-LUZ 23, 54-58.

Statistical analysis system institute (SAS). SAS/STAT User's Guide (Release 9.2). SAS Inst. Inc., Cary NC, USA.

Steverink DW, NM Soede, GJ Groenland, FW van Schie, JP Noordhuizen, B Kemp. 1999. Duration of estrus in relation to reproduction results in pigs on commercial farms. J Anim Sci 77, 801-809.

Suriyasomboon A, N Lundeheim, A Kunavongkrit, S Einarsson. 2006. Effect of temperature and humidity on reproductive performance of crossbred sows in Thailand. Theriogenology 65, 606-628.

Tantasuparuk W, N Lundeheim, AM Dalin, A Kunavongkrit, S Einarsson. 2000. Reproductive performance of purebred Landrace and Yorkshire sows in Thailand with special reference to seasonal influence and parity number. Theriogenology 54, 481-496.

Tantasuparuk, W, AM Dalin, N Lundeheim, A Kunavongkrit, S Einarsson. 2001. Body weight loss during lactation and its influence on weaning-to-service interval and ovulation rate in Landrace and Yorkshire sows in the tropical environment of Thailand. Anim Reprod Sci 65, 273-281.

Tummaruk P, N Lundeheim, S Einarsson, AM Dalin. 2000. Reproductive performance of purebred swedish landrace and swedish yorkshire sows: II. Effect of mating type, weaningto-first-service interval and lactation length. Acta Agric Scand Seet A Anim Sci 50, 217-224.

Vogrin B, D Škorjanc. 2008. Factors influencing on post-weaning performance of primiparous and multiparous sows: a review. Agricultura 6, 5-12. 
